\title{
Processamento de conteúdo, proficiências, deficiências e de interações sociais para a preparação para o Exame Nacional do Ensino Médio
}

\author{
Marcos Arrais $^{1}$, Jonice Oliveira (orientadora) ${ }^{2}$ \\ ${ }^{1}$ Instituto de Ciências Exatas e Informática - Pontifícia Universidade Católica de Minas \\ Gerais (PUC Minas) - Belo Horizonte, MG - Brazil \\ ${ }^{2}$ Programa de Pós-graduação em Informática, Universidade Federal do Rio de Janeiro - \\ Rio de Janeiro, RJ - Brazil \\ arraisepucminas.br, jonice@dcc.ufrj.br

\begin{abstract}
Thousands of students in Brazil need studies complementary to formal education to achieve good scores in the national high school examination (Exame Nacional do Ensino Médio - ENEM) and guarantee entry into higher education. This work aims to develop a method to measure the proficiencies and deficiencies of students and guide them in the construction of new knowledge. The developed artifact is an adaptive tool, whose engagement is based on gamification concepts. The methodology used was Design Science Research. The evaluation of the artifact was made through a Case Study and the results demonstrate the effectiveness of the proposed method.
\end{abstract}

Resumo. Milhares de estudantes no Brasil necessitam de estudos complementares ao ensino formal para atingir boas pontuações no Exame Nacional do Ensino Médio - ENEM e garantir o ingresso no ensino superior. Esse trabalho tem por objetivo o desenvolvimento de um método para mensurar as proficiências e deficiências dos alunos e orientá-los na construção de novos conhecimentos. $O$ artefato desenvolvido é uma ferramenta adaptativa, cujo engajamento é baseado em conceitos de gamificação. A metodologia utilizada foi Design Science Research. A avaliação do artefato foi feita através de Estudo de Caso e os resultados demonstram a efetividade do método proposto.

\section{Visão Geral do Tema}

No Brasil, a escola pública muitas vezes sofre pela falta de investimentos, infraestrutura e corpo docente, e segundo Moraes (2014) pode apresentar uma disparidade de até $120 \%$ em indicadores de qualidade de ensino quando comparadas com escolas privadas. O grande problema é que os alunos formados na escola pública e privada passam pelo mesmo processo de ingresso ao ensino superior.

Existem inúmeras iniciativas livres na internet que apoiam o ensino formal com técnicas de auto-aprendizagem, sejam através de plataformas de simulados, videoaulas ou mesmo discussões em um grupo aberto de uma rede social on-line. A grande dificuldade dessas plataformas é a ausência de um método avaliativo que possa aferir o crescimento, erro, acerto e carência de parte do conteúdo programático. Muitas vezes quando a aluno inicia um processo de estudo informal, ele não sabe por onde começar e 
muitas vezes salta unidades formativas que tem pré-requisito. Esse processo ocorre em mecanismos de aprendizagem sem nenhuma mediação e avaliação.

Essa carência da rede pública de ensino deve ser suprida para garantir uma igualdade de acesso ao ensino superior. Estudantes de baixa renda muitas vezes não podem pagar por um pré-vestibular e tem na internet uma fonte livre para complementar seus estudos. Esse estudo informal é apoiado por navegação em sites de conteúdo, fóruns e rede social, ao qual podemos destacar o Facebook como uma importante ferramenta de estudos.

Nesse contexto, este estudo objetiva a formulação de um método de autoaprendizagem, focado na preparação de estudantes para o Exame Nacional do Ensino Médio - ENEM. Para validar e aferir a eficiência do método proposto a pesquisa construiu um subproduto, um artefato ${ }^{1}$ web-mobile que utiliza dados públicos da rede social online Facebook para classificar os conteúdos a serem estudados com base nas proficiências e deficiências do aluno. O sistema web possui uma camada de gamificação, onde na visão de Zichermann (2011) essa estratégia envolve e engaja o aluno nas tarefas necessárias para que a ferramenta colete os dados para inferir os conteúdos a serem estudados.

Entendendo o trabalho como uma contribuição ao desenvolvimento do campo de Sistemas de Informação e aparado pelo relatório técnico de Araújo et al., (2017), que retrata os grandes desafios da área para um horizonte de 2016 a 2026, entende-se que essa pesquisa se enquadra no tópico de "Sistemas de Informação no Mundo Aberto". Em um dos trabalhos do relatório Araújo (2016) descreve que "em um período no qual vivenciamos uma disrupção tecnológica pela convergência da colaboração, mobilidade e grande volume de dados, o desafio para a pesquisa em SI está em como promover a integração destas tecnologias para balancear tanto as necessidades de controle como as oportunidades do comportamento emergente e sua inovação.”. O trabalho relata ainda a necessidade de construção de sistemas para a resolução de problemas complexos da humanidade e no presente estudo, o artefato proposto tem por objetivo ajudar estudantes a desenvolverem o conhecimento acerca dos conteúdos do ENEM. Além disso, este trabalho também está levemente alinhado com o desafio "Metodologias e tecnologias para participação popular". Embora esta tese não esteja ligada diretamente ao conceito padrão de e-Democracia, os artefatos desenvolvidos (método e ferramenta) auxiliam no engajamento e participação dos estudantes para a criação de conhecimento. Além do engajamento, a participação social visa homogeneizar o conhecimento e permitir que alunos (pertencentes a categorias com histórico de baixa aprovação e inserção nas melhores universidades brasileiras) tenham maiores chances de sucesso. Consequentemente, estamos trabalhando com tecnologias para a participação social que terão um impacto na mobilidade social, através do estudo. Tais princípios (acesso à informação, criação de conhecimento e mobilidade social) estão diretamente ligados à Democracia.

\section{Definição dos Objetivos e Relevância da Pesquisa}

Essa pesquisa tem por objetivo geral o desenvolvimento de um método automatizado de estudo informal baseado em redes sociais que aprimore o processo de autoaprendizagem

\footnotetext{
${ }^{1}$ Demonstração do artefato desenvolvido: Plataforma ENEM na Rede - https://www.youtube.com/watch?v=4pyhTwEFjMY
} 
na preparação para o ENEM. Os objetivos específicos decompõem o escopo geral da pesquisa e foram definidos em: mapeamento sistemático; proposição de artefato para resolução do problema; criação de procedimentos para coleta, mineração dos conteúdos e identificação das habilidades no formato de questões objetivas; avaliação com público real para aferir a eficiência do método proposto; coleta e análise dos dados do cenário de avaliação; publicação dos resultados completos.

No tocante à relevância do estudo, destaca-se o viés inédito e exclusivo do trabalho. Como principal contribuição científica, podemos destacar a criação de um método capaz de mensurar proficiências e deficiências pessoais em áreas do conhecimento, bem como estratégias para diminuir esta diferença. O método desenvolvido integra conceitos de recuperação da informação, mineração de dados, workflow, representação da informação e gamificação. Como contribuição técnica podemos destacar a ferramenta, a qual está disponibilizada e continua em uso por alunos da rede pública. Como contribuição social, podemos destacar um recurso público e gratuito para auxiliar estudantes que estão em preparação para exames como o ENEM. Fora isso, toda a solução pode ser apropriada por pesquisadores para a construção de ferramentas livres, que se apoiem no poder da Internet para ajudar alunos no processo de construção do conhecimento.

\section{Trabalhos Relacionados}

O processo de revisão de literatura dessa pesquisa foi substanciado pelo desenvolvimento de uma revisão sistemática, orientada pelas questões principal e secundária do estudo, que são definidas como:

- Questão principal: Como coletar deficiências de estudo de usuários em uma rede social on-line?

- Questão secundária: Como formular um método de estudo informal que sequencie deficiências e gere um plano de estudos ao usuário?

No mapeamento sistemático foram encontrados um total de 473 publicações nas consultas às bases de pesquisas. Na etapa de seleção primária, onde são realizadas as leituras de título e resumo (abstract) foram selecionados: 72 para a questão principal, 18 para a questão secundária e 2 para a questão principal e secundária. $\mathrm{Na}$ etapa final de seleção é feita a sumarização de resultados com a leitura completa da pesquisa. Nessa etapa foram selecionados: 20 estudos para a questão principal, 10 para a questão secundária e 7 que atendiam a ambas.

Um número pequeno de trabalhos representa a área de recuperação da informação com técnicas de scraping combinado a utilização de API pública, como é o caso do estudo de Cox et al. (2011) que desenvolveu padrões de métricas para caracterizar grupos do Flickr. Na área de processamento de linguagem natural destacase o trabalho de Herdağdelen e Baroni (2011) para identificação de gênero em redes sociais on-line, que utiliza uma base gramatical para comparações. Alguns trabalhos usavam serviços ou abordagens de terceiros para o funcionamento do método proposto, como por exemplo a utilização do PageRank e SentiWordNet para cálculo de polaridade 
de tweets (Montejo-Ráez et al., 2014) e a construção de querys de consulta por palavraschaves que eram aplicados diretamente em motores como o Google Search (Zhou e Pei, 2011).

Com temáticas de classificação e recomendação alguns trabalhos se destacam, como por exemplo, o algoritmo para recomendações em sistemas de busca (Shapira e Zabar, 2011), um framework para geração de rótulos de classificação em redes sociais, chamado de SocialDim (Tang e Liu, 2011), extração automatizada de conteúdo por tópicos (Kaptein e Kamps, 2011), processamento léxico e sintático na classificação de polaridade de mensagens (Vilares et al., 2015) e o cluster de usuários baseado em interações em quadro de mensagens (Hernández-García e González-González, 2015).

Abaixo alguns estudos também selecionados na revisão sistemática:

1. Extração e análise de opiniões através de sistema de lógica fuzzy (Kaiser e Bodendorf, 2012);

2. Inferência de aprendizagem através do conceito de matriz de fatoração, introduzindo a medida para cálculo de similaridade de usuários e itens, definida como meta-path (Shi et al., 2016);

3. Como coletar dados de inteligência coletiva para gerar marcações sociais. O método OverlapRank apresentado pode ser utilizado como fator de ordenação (Yi, 2012);

4. Testar hipóteses sobre a eficiência, qualidade e significância de respostas em comunidades de conhecimento. (Kang et al., 2011).

Alguns trabalhos conhecidos não foram identificados na revisão sistemática por serem trabalhos não científicos, sem publicações nos principais veículos. Como estão relacionados ao tema, incluímos para fins de comparação. São eles: portal Descomplica.com.br e a plataforma "Hora do Enem" do Ministério da Educação.

É possível observar que no panorama de trabalhos correlatos apresentado, cada estudo cobre somente uma parte do problema de pesquisa, não existindo, no universo amostral selecionado, um trabalho que solucione as questões principal e secundária da pesquisa. A revisão de literatura aponta técnicas, métodos e abordagens que somados podem ter significativa importância a essa pesquisa, mas que isoladamente resolvem problemas em seus contextos específicos.

Mesmo trabalhando um amplo contexto a pesquisa expõe como a ciência pode evoluir um campo utilizando abordagens de sucesso de outros cenários de forma combinada. Pelos resultados apresentados com o uso do artefato proposto podemos estabelecer novas fronteiras para o uso de tutores inteligentes para a educação, expandindo o horizonte da sala de aula e entregando mais autonomia a estudantes.

\section{Métodos Científicos - Design Science Research}

A metodologia utilizada na pesquisa foi o Design Science Research (DSR) descrita nos trabalhos de Dresch et al. (2015). A DSR sistematiza a construção de artefatos - que podem ser softwares, por exemplo, orientados por conjecturas teóricas, advindas da revisão de literatura e sistemática, que dão suporte a resolução de problemas. $\mathrm{O}$ processo é descrito em ciclos e as iterações melhoram o artefato podendo incorporar novas pesquisas/conceitos. Deve ser lembrado também que tal metodologia privilegia a 
construção de conceitos e artefatos generalistas, que possam ser utilizados em outros contextos, gerando uma maior integração entre teoria e prática.

Aplicando a metodologia a primeira fase da pesquisa concentrou-se em definir o problema. Essa definição foi essencial para a condução da revisão de literatura. Com o problema decomposto nas questões de pesquisa a segunda parte da metodologia foi a análise e diagnóstico, que foi importante para perceber as correlações entre os trabalhos selecionados. Com os conceitos passa-se para a etapa de desenvolvimento, que no caso do estudo, começou com o desenho arquitetural da proposta, passando pela implementação do algoritmo principal de proficiências e deficiências, os robôs autônomos e por fim as camadas de front-end ${ }^{2}$, para que os usuários pudessem interagir com a plataforma. Por fim foi conduzido um teste de adoção da plataforma para verificar se as métricas fornecidas estavam dentro dos pressupostos da pesquisa. Ela consistiu na aplicação de simulados no formato ENEM para dois grupos distintos de alunos do ensino médio. O primeiro simulado foi aplicado no início do processo de testes, para aferir o estado real e atual de conhecimento que o estudante se encontrava, após esse teste os alunos iniciavam o uso livre da plataforma, somente com as instruções e tutoriais de uso que estavam contidos internamente. O segundo grupo após a aplicação do simulado era liberado para continuar seus estudos domiciliares, mas não utilizava a plataforma. Depois do período de três meses, os dois grupos refaziam um novo simulado e as métricas de evolução eram comparadas.

Seguindo a abordagem proposta por Dresh et al. (2015) foram estruturadas as atividades que seriam realizadas em cada um dos doze elementos descritos no estudo. A Figura 1 detalha cada etapa e as entregas realizadas.

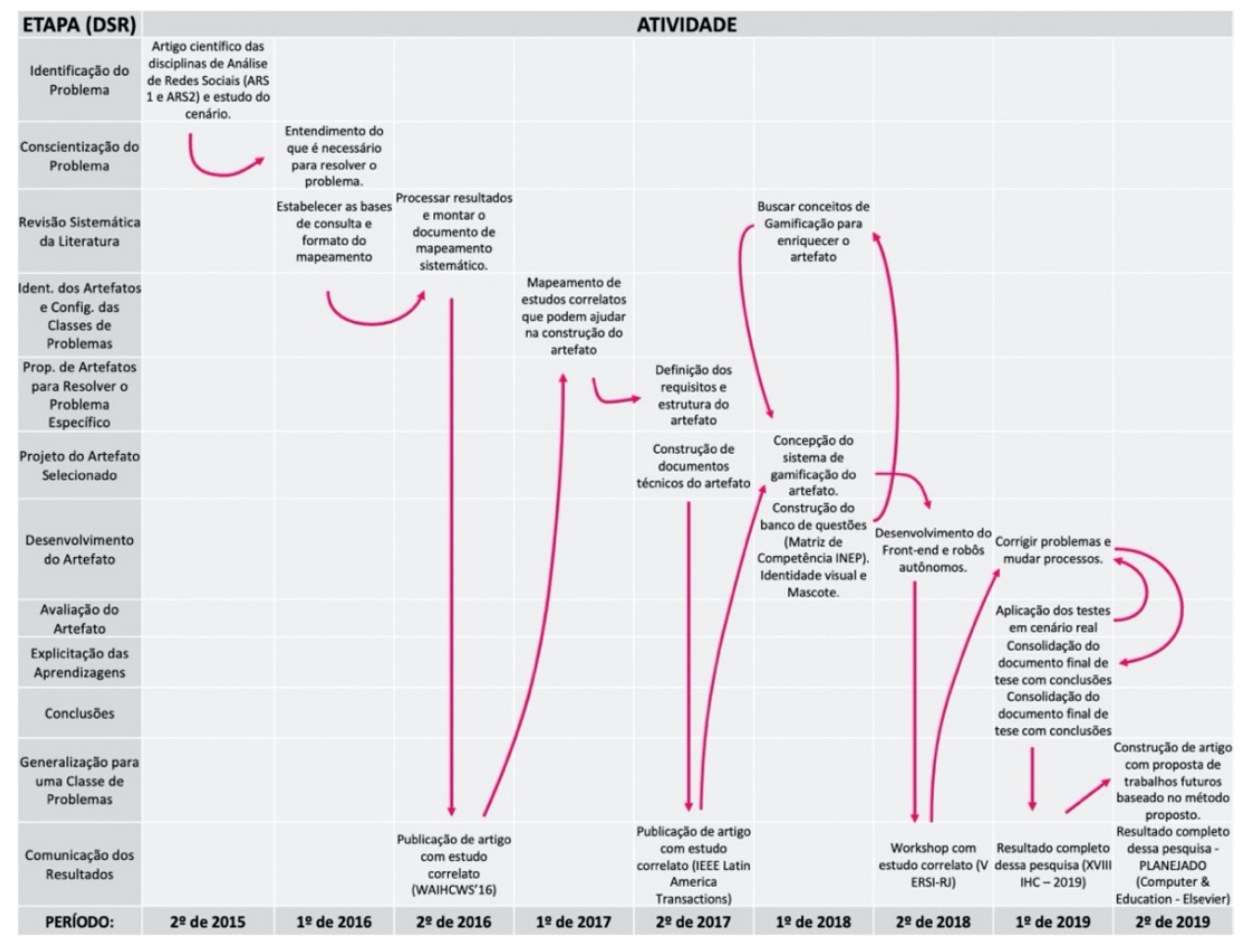

Figura 1: Ciclos e entregas da pesquisa baseado no método de Design Science Research de Dresh et al. (2015) - Fonte: adaptado pelo autor de ARRAIS (2019).

\footnotetext{
${ }^{2} \mathrm{O}$ front-end é responsável por coletar a entrada do usuário em várias formas e processá-la para adequá-la a uma especificação em que o software do servidor possa utilizar.
} 
É importante ressaltar que a fase de identificação do problema, fornecido pelo DSR, deu a pesquisa as dimensões que deveriam ser trabalhadas pelo método proposto, como por exemplo:

- A dificuldade de os alunos gerenciarem dependências de conteúdos;

- Mensurar o crescimento da sua aprendizagem;

- Socializar o estudo do aluno gerando coletivos que estão agrupados em torno de um contexto comum (a preparação para o ENEM);

- Mostrar qual estudo deve ser priorizado e qual o aluno é proficiente, podendo concentrar seus esforços em uma área deficitária.

\section{Desenvolvimento do Método e Artefato}

Para garantir significativos ganhos de aprendizagem dos conteúdos das quatro grandes áreas do ENEM (linguagens e códigos, matemática, ciências humanas e da natureza), foi elencada uma estrutura de funcionamento da ferramenta que está definida em: i) gerar testes com questões no padrão ENEM; ii) avaliar os erros/acertos dos testes e definir proficiências e deficiências de conteúdos; iii) montar um plano de estudos individualizado com as necessidades de conteúdos do aluno; iv) oferecer conteúdos de repositórios externos para que o aluno utilize como apoio no processo de estudo; v) reiniciar esse processo de forma continua.

A primeira fase do desenvolvimento foi à mineração de questões de domínio público do ENEM de anos anteriores e de universidades de todo o Brasil para compor a base de perguntas que seriam utilizadas para validar os conhecimentos do aluno. Essa foi uma das etapas mais importantes porque todo conteúdo adicionado ao banco de dados necessitava de uma adequação para encaixar com a matriz de competências do ENEM. Ao todo foram mapeadas 32 mil questões. Com a interface gráfica, finalizada e regras de negócio implementadas, deu-se início a construção dos robôs autônomos que funcionam como serviços da ferramenta. Tais agentes autônomos são responsáveis por coletar os conteúdos utilizados na plataforma e realizar rotinas autônomas em intervalos de tempo definidos, como por exemplo, calcular as proficiências e deficiências de alunos, além de extrair dados relevantes da rede social do aluno.

\subsection{Funcionalidades da Ferramenta}

O funcionamento da ferramenta está dividido em quatro requisitos. São eles:

- Batalha: principal área da plataforma. Aqui são apresentadas as questões no formato do ENEM e a partir dos erros e acertos dos alunos, o sistema calcula as proficiências e deficiências para construir um plano de estudo para o aluno. Essa área da plataforma é executada em duplas, onde um estudante convida outro para responder uma rodada de perguntas;

- Sugestão de Estudos: é o campo onde são apresentados os planos de estudos do aluno. O sistema seleciona alguns assuntos relacionados às deficiências identificadas durante as batalhas. Após a revisão desses assuntos, o conteúdo é inserido novamente como batalha para que seja avaliado se houve o aprendizado;

- Grupo de Estudos: coleta as interações sociais dos estudantes em um grupo público de Facebook e analisa seu conteúdo. Caso os assuntos debatidos sejam 
pertinentes ao ENEM, a plataforma atribui pontos ao estudante no sistema de gamificação;

- Ranking: é onde o estudante pode ver seus pontos e sua colocação no sistema. Todas as atividades da ferramenta são pontuadas. Essa mecânica é responsável pelo maior envolvimento do aluno uma vez que suas conquistas são compartilhadas com outros usuários da rede.

A Figura 2 demonstra a interface final da ferramenta proposta:

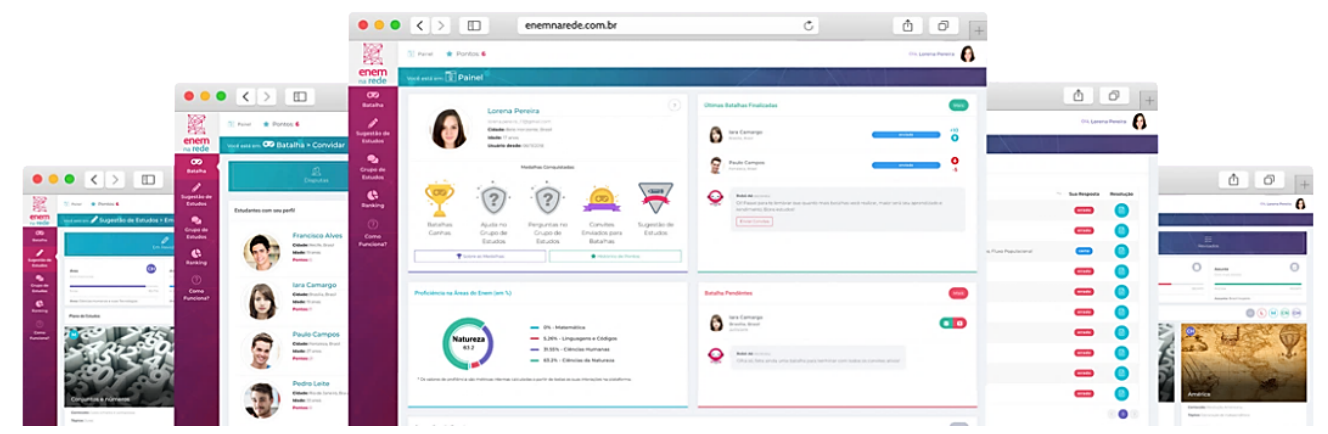

Figura 2: Interface da ferramenta - Enem na Rede. Fonte: ARRAIS (2019).

\subsection{Arquitetura e Tecnologias da Plataforma}

A figura 3 demonstra a arquitetura conceitual da plataforma e mostra como os serviços e abordagens web estão interconectados para a construção de um artefato único. É importante observar que toda a interação do aluno acontece em um front-end web que usa os dados de usuário fornecidos pela plataforma Facebook.
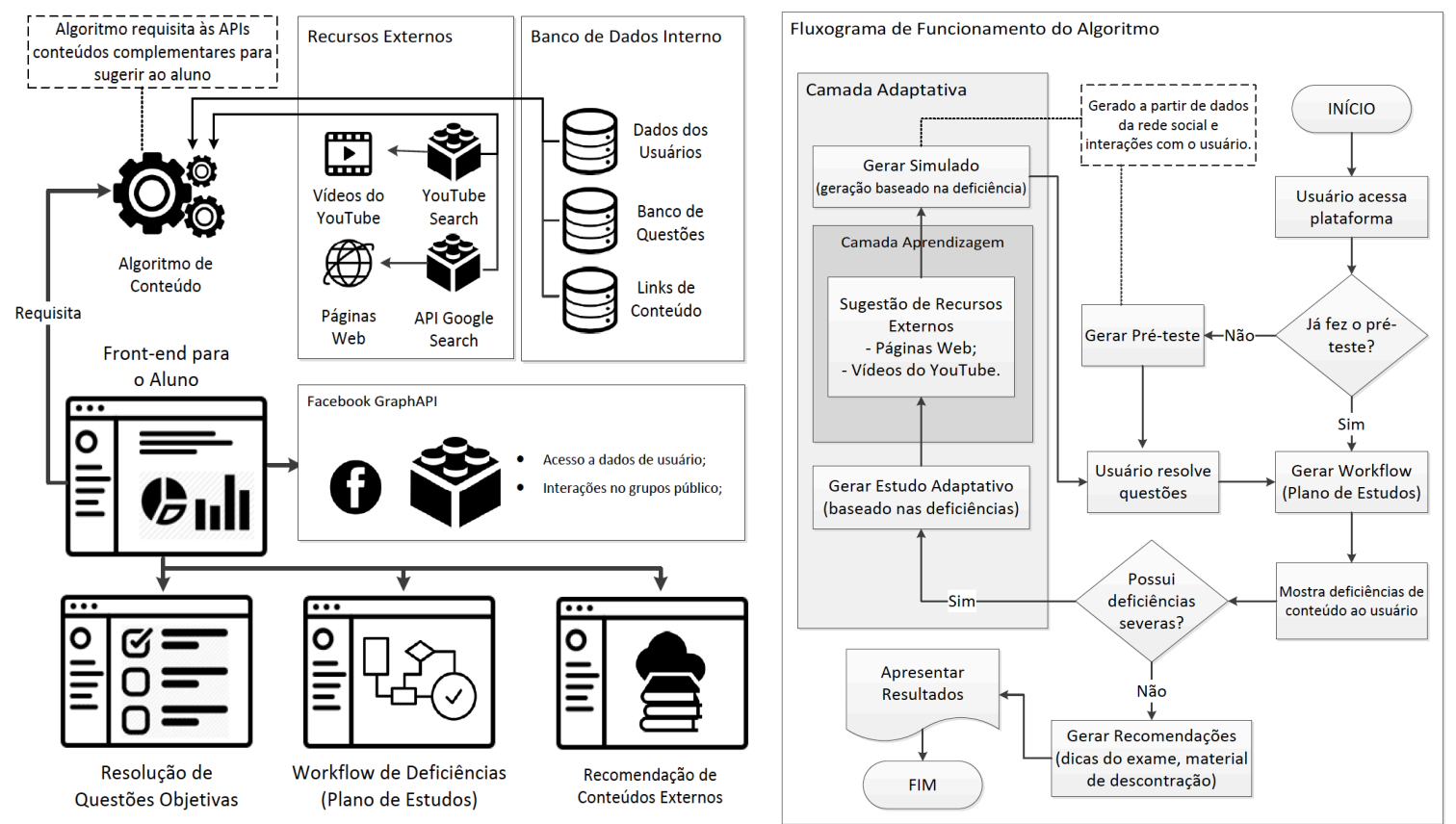

Figura 3: Proposta de Arquitetura da Plataforma. Fonte: ARRAIS (2019).

\subsection{Processador de Conteúdos}

É responsável por percorrer todo o banco de questões e para cada questão minerar através da Google Search API e o do Youtube Search API conteúdos que atendiam a 
critérios pré-definidos. O robô escolhe os conteúdos que irão compor a base referenciado na reputação atribuída pelas search engines. No caso da Youtube Search também são considerados as curtidas e inscrições que o vídeo e canal possuem. É importante ressaltar que somente conteúdos públicos da internet são indexados pelos robôs.

A abordagem para coleta de websites aproveita a inteligência do algoritmo de pagerank do Google Search. Sendo assim, o robô utilizando a API pública Google Search API extrai os resultados e classifica quais irão para a base. O termo de consulta é obtido através da extração das palavras-chave da questão mais a combinação do assunto, tópico e subtópico da questão. A extração da palavra-chave utiliza o seguinte procedimento: $1^{\circ}$ - remoção de stop words; $2^{\circ}$ - stemming e lowercase $; 3^{\circ}$ - contagem da frequência do termo; $4^{\circ}$ - seleção dos três primeiros termos. Para consolidar a query de busca são utilizados operadores booleanos e aspas para enclausurar expressões. Seguindo a estrutura: "assunto" AND "tópico" AND "subtopico" AND ("palavra-chave 1" OR "palavra-chave N").

Durante o processo de extração de dados via API o robô executa também o processo de classificação dos itens. Para que os endereços de website entregues pela Google Search API sejam selecionados, eles devem atender os seguintes critérios:

- O conteúdo deve ter mais de um ano que figura nos resultados de busca;

- Devem estar em português;

- Obrigatório conter o assunto e tópico no corpo do texto.

Para os vídeos selecionados da plataforma Youtube:

- O saldo de curtidas (likes) deve ser maior que o de não-curtidas (unlikes);

- Devem existir comentários no vídeo;

- Deve ter um canal com outros vídeos associados.

É importante ressaltar que em ambas as consultas é respeitado o ranking que o sistema de busca classificou. Sempre que o número mínimo de resultados ( 2 websites e 2 vídeos) não é atingido, o robô refaz a busca removendo subtópico e depois tópico. Se depois de remover subtópico e tópico as quantidades mínimas não forem cumpridas, o algoritmo cadastra a quantidade encontrada e envia um e-mail para o administrador do sistema com a query de busca e o código da questão para que o processo seja realizado manualmente.

\subsection{Processador de Proficiências e Deficiências}

Esse robô é responsável por processar os conteúdos que o aluno tem deficiências e sugerir um plano de estudos a ele. Sua instância é lançada sempre que um aluno realiza um teste dentro da plataforma. A parte fundamental do processo está em somar ao final o grau de dificuldade do item $\left(D_{i}\right)$ estabelecido por Pasquali (2003) como:

$$
D_{i}=\frac{C_{i}-\frac{E_{i}}{K_{i}-1}}{N_{i}}
$$

\section{Equação 1 - Nível de dificuldade com influência da escolha aleatória por} Pasquali (2003) 
Onde a dificuldade de um item $\left(D_{i}\right)$ é representada pelo número de indivíduos que responderam corretamente um item $\left(C_{i}\right)$ menos a razão entre o número de alunos que erraram o item $\left(E_{i}\right)$ pelo número de respostas do item $\left(K_{i}\right)$ menos um, dividido pelo número de indivíduos que realizaram o teste $\left(N_{i}\right)$.

Baseado nos erros/acertos do teste, o robô utiliza o algoritmo de proficiência/deficiência para aferir quais conteúdos demandam atenção.

Quando um aluno acerta uma questão, é necessário invocar os graus de proficiência históricos para aquele tópico e subtópico de conteúdo (Valores de Proficiência - $V_{p}$, no qual $\boldsymbol{n}$ é o total de registros da base, para aquele tópico e subtópico), que estão gravados na base de dados.

Sendo assim para a equação de proficiência (Arrais, 2019) temos:

$$
\text { Proficiência }=\log \left(\frac{\sum_{i=0}^{n} V_{p}+1}{\sum_{i=0}^{n} V_{d}+1}\right)+D_{i}
$$

\section{Equação 2 - Cálculo de proficiência em um tópico específico de conteúdo. Fonte: ARRAIS (2019).}

$\mathrm{Na}$ equação de deficiência (Arrais, 2019), o grau de dificuldade da questão tem um peso maior do que na proficiência. Essa adequação faz com que o estudante tenha que acertar mais de uma questão para que seu grau seja convertido de deficiência em proficiência.

$$
\text { Deficiência }=\log \left(\frac{\sum_{i=0}^{n} V_{d}+1}{\sum_{i=0}^{n} V_{p}+1}\right)+1+D_{i}
$$

\section{Equação 3 - Cálculo de deficiência em um tópico específico de conteúdo. Fonte: ARRAIS (2019).}

Quando uma questão não possui a dificuldade $\left(D_{i}\right)$ calculada, por ainda não ter uma base de respondentes de 100 usuários o coeficiente de dificuldade é alterado para o valor 1 de deficiência e 0.5 quando se tratar de proficiência. O coeficiente de dificuldade $\left(D_{i}\right)$ é recalculado sempre que um teste é processado, mantendo o equilíbrio da base, visto os novos usuários e testes que são realizados durante a execução do artefato.

Os valores de proficiência e deficiência são utilizados para definir quais conteúdos devem ser apresentados no workflow. Quanto maior a deficiência, maior a prioridade no ranking de conteúdos. Quando o saldo de proficiência é maior que o de deficiência o conteúdo é transferido para uma área de itens revisados.

\subsection{Processador de Interações Sociais}

Responsável por quantificar a participação do aluno no grupo de estudos da plataforma. O grupo de estudos opera dentro da rede social on-line (RSO) Facebook e, diariamente, a cada 2 horas, o robô visita a rede, extrai as participações do aluno e verifica se elas têm correlação com algum conteúdo da base. Caso positivo o aluno é bonificado com uma quantidade de pontos no sistema de gamificação da plataforma. 
Para processar as interações dos alunos no grupo, diariamente a cada duas horas um robô conecta-se a Graph API do Facebook e lê todas as postagens realizadas no grupo. As postagens são divididas em dois grupos: perguntas e respostas. As perguntas são postagens realizadas no fluxo principal do grupo, conhecido como feed. As respostas são comentários adicionados a perguntas do feed. Para cada post realizado, o algoritmo verifica qual é o autor do conteúdo e processa o texto para identificar se está relacionado a algum conteúdo da matriz de referência do ENEM. Caso positivo, ele salva o conteúdo na área de "Grupo de Estudos" do aluno e atribui pontos pela publicação.

O processamento do conteúdo é realizado por técnicas de recuperação da informação, que extrai do texto do aluno as palavras-chave e compara com a base de dados de questões do artefato. A primeira fase de construção do algoritmo foi à indexação de todas as palavras-chave das questões, agrupadas por subtópico e tópico. Sempre que um novo conteúdo é recuperado, suas palavras-chave são comparadas às palavras-chave da base indexada. Esse processo retorna um coeficiente de correlação: Quanto mais alto, maior a probabilidade de os conteúdos serem relacionados. Para a postagem do aluno, os tratamentos realizados nas strings foram: $1^{\circ}$ - remoção de stop words; $2^{\circ}$ - stemming e lowercase; $3^{\circ}$ - contagem da frequência do termo. Já para o processo de indexação, que é usado como a base de comparação para a primeira fase, foram considerados os seguintes passos:

- Junção de todas as palavras-chave das questões por tópico;

- Remoção das strings duplicadas para o atributo palavras-chave, stop words, stemming e lowercase;

- Junção de todos os subtópicos das questões por tópico;

- Remoção das strings duplicadas para o atributo subtópico, stop words, stemming e lowercase;

- Agrupamento por tópico e assunto;

- Indexação dos termos utilizando o método Term frequency-inverse document frequency selection (TF-IDF) - Leginus, 2015;

Após o processo de indexação, o robô compara se o texto digitado pelo aluno tem correlação com os textos indexados na base, retornando um coeficiente.

Percebeu-se que somente aplicar o método TF-IDF padrão não geraria uma correlação funcional. Foi então que o algoritmo foi adaptado para funcionar com pesos individuais em cada atributo (tópico, subtópico e palavra-chave). Além de acrescentar o peso individual foi adicionado um fator de normalização do TF que utiliza o tamanho do documento como um critério de importância. Essa normalização garantiu melhorias significativas da correlação. Enquanto alguns documentos indexados possuem poucas palavras-chave, visto o baixo número de questões relacionadas na base, outros documentos possuem muitas questões relacionadas, resultando em um grande número de palavras-chave e consequentemente no aumento do tamanho do documento. $\mathrm{O}$ processo de normalização permitiu que os documentos com tamanhos diferentes pudessem ser comparados. A equação final foi definida matem: 


$$
\begin{gathered}
T F-I D F \text { Normalizado }=T F * I D F \\
T F=\sqrt{T F_{x, y}} * \frac{1}{\sqrt{l}} * w \\
I D F=\left(\log \frac{N+1}{d f+1}+1\right) * w
\end{gathered}
$$

\section{Equação 4 - Adaptação do TD-IDF para uso de pesos variáveis e normalização. Fonte: ARRAIS (2019).}

Onde $T F_{x, y}$ é a frequência de uma palavra-chave $X$ em um documento $Y, N$ é o total de documentos na base, e $d f$ é o número de documentos que contém a palavrachave $X, \mathrm{o} w$ é o peso que será variável por atributo, trazendo a possibilidade de definir uma maior importância, por exemplo ao subtópico. O $l$ é o tamanho do documento em palavras.

Os documentos são indexados e seus atributos receberam os seguintes fatores de importância: topico $_{w}=6 ;$ subtopico $_{w}=4 ;$ palavras - chaves $_{w}=0.6$. Esse coeficiente foi ajustado realizando vários testes para processar a eficiência. É possível observar que se uma string está presente no tópico do documento, ela ganha um alto fator de importância, o que faz sua nota de correlação cresça.

\subsection{Tutoria do aluno mediado por agente}

Durante a construção das interfaces observou-se que o artefato precisava dialogar constantemente com o usuário, informando o término de atividades, instruindo sobre qual conteúdo ele deveria visitar, quais conteúdos foram importados no "Grupo de Estudos". Esse diálogo contínuo trouxe a necessidade de criar uma representação visual que mediasse à comunicação do artefato com o aluno. Assim surgiu o tutor da plataforma ENEM na Rede, que recebeu o nome de "Aê", que em tupi-guarani significa "Amigo". Como o funcionamento do artefato já era baseado na operação de três serviços automatizados, os robôs de conteúdo, proficiência/deficiência e interações sociais, o tutor recebeu a identidade de um robô, com traços simples e utilizando as cores do artefato. A figura 3 ilustra algumas situações de diálogo entre o "Aê" e o aluno.

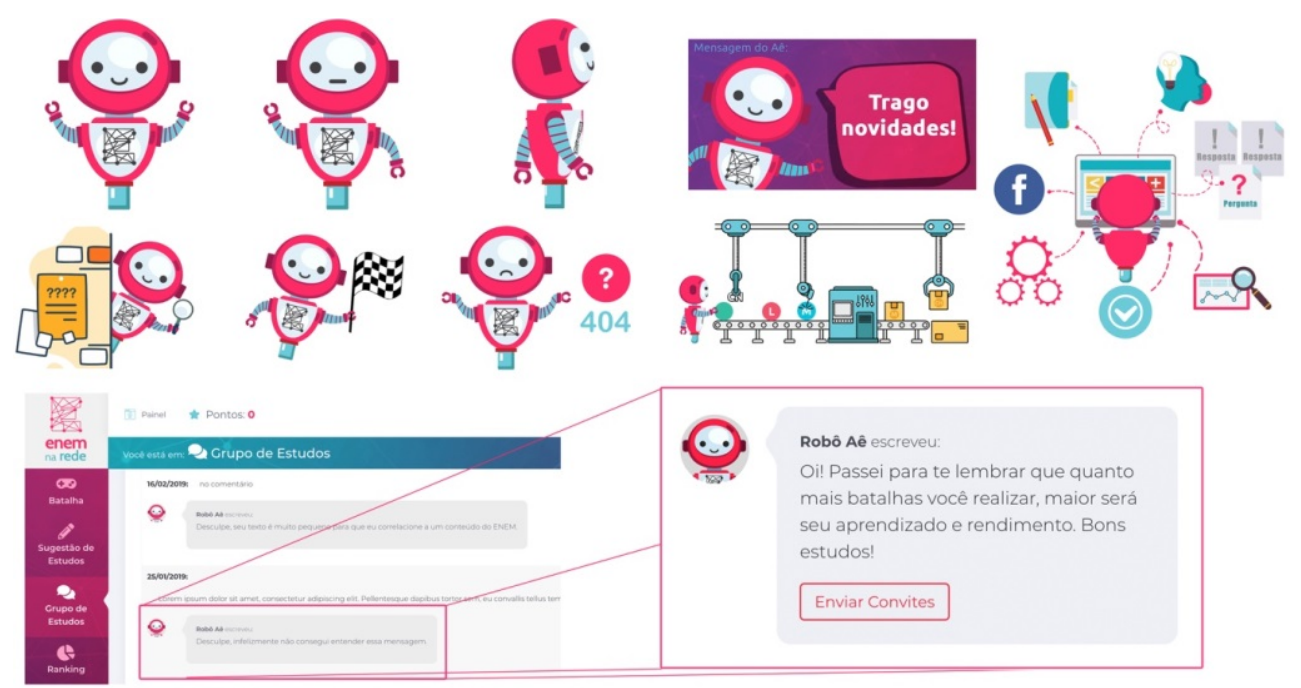

Figura 4 - llustrações do mascote "Aê" na plataforma ENEM na Rede. Fonte: ARRAIS (2019). 


\subsection{Gamificação}

Foi observado que o processo cíclico e contínuo das atividades do artefato poderia não gerar muito engajamento e participação por parte dos alunos, desmotivando o uso do artefato, uma vez que o processo de operação ficava restrito a: responder questão; acessar conteúdos; repetir o processo. Observou-se então a necessidade de uma técnica mais envolvente para o uso do sistema, a assim as dinâmicas de gamificação foram incorporadas ao projeto, gerando duas mecânicas sociais para envolver o aluno no uso do artefato, são elas: testes baseados em batalhas entre estudantes e um sistema de pontos com medalhas (badges) para premiação baseado em participação.

$\mathrm{Na}$ primeira abordagem o aluno ao invés de responder um teste de forma individual e que está relacionado somente a suas proficiências/deficiências, agora ele realiza em dupla com outro estudante que tem um perfil semelhante ao seu. Essa mecânica social cumpre um dos pressupostos básicos da Gamificação, o desafio. Nesse modelo os testes passam a se chamar "batalhas" e o estudante para realizá-las deve fazer o convite a outro estudante, que deve aceitar ou recusar. Ao aceitar uma batalha o sistema irá gerar um teste que contempla a necessidade do aluno e do seu oponente. $\mathrm{O}$ teste é composto por dez questões fechadas que trabalham as quatro grandes áreas do ENEM. O número de questões foi definido tendo em vista que o tempo médio de execução da batalha não excedesse 15 minutos, um teste muito longo poderia desmotivar o aluno e gerar muitos itens de conteúdo para revisão.

No segundo mecanismo proposto, foi definido que todas as interações dentro do artefato gerariam pontos, que seriam utilizados para classificar os estudantes e mostrar a pontuação em seu perfil. Já o sistema de medalhas premia o estudante com uma insígnia (badge) baseado na quantidade de atividades que ele realizou, as atividades são: batalhas, ajuda no grupo de estudos, perguntas no grupo de estudo, convite de batalhas e sugestão de estudos realizadas. A tabela 1 demonstra as atividades necessárias para conquistar uma medalha e apresenta as evoluções possíveis dentro do artefato.

Tabela 1. Medalhas (Badges) que podem ser conquistados na plataforma. Fonte: ARRAIS (2019).

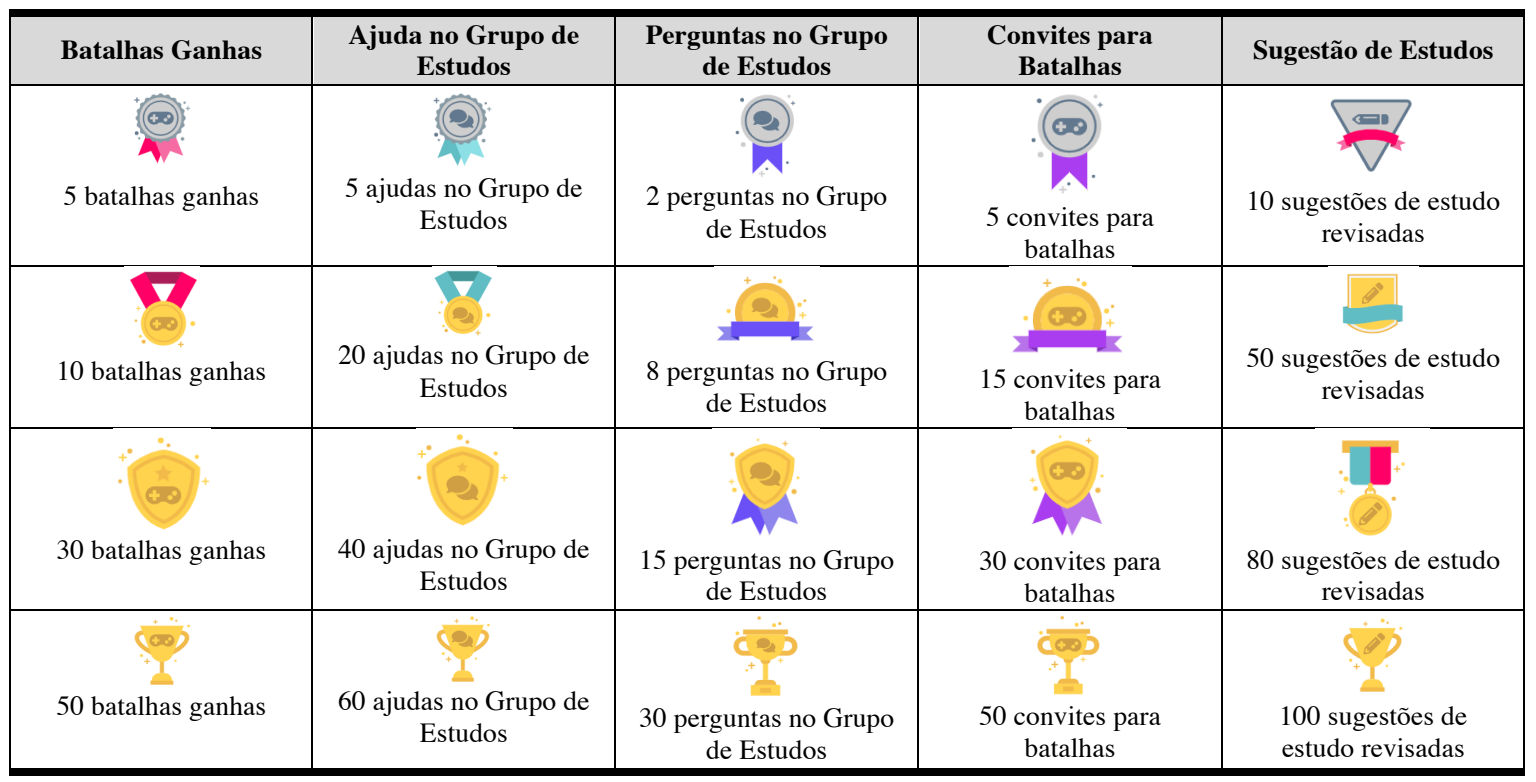




\section{Coleta de Dados e Análise de Resultados}

O pesquisador realizou uma consulta a escolas da rede pública e privada da cidade de Belo Horizonte - MG para verificar se existia interesse em participar dos testes de validação do método e artefato. As consultas foram realizadas no período de 12/02 a 27/02 de 2019. Por se tratar de um teste extenso, muitas escolas visitadas apresentaram o problema de conciliação das atividades com o calendário acadêmico.

As etapas do experimento foram: seleção; divisão de grupos; aplicação do Simulado ENEM (fase 1); vivência na plataforma; aplicação do Simulado ENEM (fase 2) e análise de resultados.

$1^{\mathbf{0}}$ Passo: Seleção - Foram selecionados 120 alunos que tinham interesse em participar do teste. Foi realizada uma aula expositiva sobre o funcionamento da plataforma e a importância dos testes de validação. $\mathrm{O}$ grupo era composto por alunos do $3^{\circ}$ ano do ensino médio do turno da noite;

$2^{\mathbf{0}}$ Passo: Divisão de Grupos - Os 120 estudantes foram divididos em 2 grupos (60 em cada). O primeiro grupo utilizaria a plataforma durante o período de três meses, entre março e junho de 2019, semanalmente de forma regular. O segundo grupo não teria acesso a plataforma e ficaria restrito ao estudo formal, de sala de aula e a estudos autônomos no ambiente domiciliar.

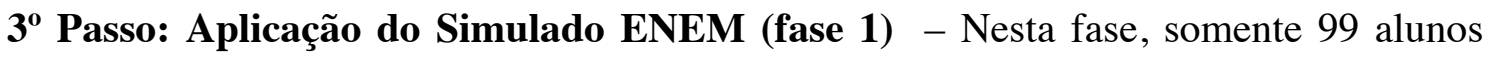
dos 120 interessados apresentaram o termo de autorização dos pais para participar do processo, sendo assim, os grupos foram divididos novamente (49/50). O processo foi aleatório, por sorteio de chaves no dia da prova. Para os dois grupos foram aplicados um simulado no padrão ENEM com 180 questões divididas nas quatro grandes áreas, para aferir o estado inicial de conhecimento.

$4^{\mathbf{0}}$ Passo: Vivência na Plataforma - Durante três meses, no período de março a maio de 2019, o grupo 1 acessou a plataforma regularmente, com monitoramento do pesquisador dos acessos diários, para se caso algum estudante não estivesse envolvido, fosse suprimido na tabulação das métricas, não interferindo na nota do restante.

$5^{0}$ Passo: Aplicação do Simulado ENEM (fase 2) - Novamente os dois grupos foram chamados para realizar um novo simulado que vai aferir a evolução deles. O grupo 1 , utilizou a plataforma, o grupo 2 ficou somente no estudo formal. Esse segundo simulado registrou um alto índice de evasão;

$6^{0}$ Passo: Análise de Resultados - Essa é a etapa de consolidação dos resultados. Os simulados são corrigidos, os dados tabulados e são traçados os perfis de evolução dos grupos 1 e 2 ;

Durante a fase de análise dos dados gerados na plataforma foi possível ainda observar outro recorte possível, baseado na utilização do artefato. O nível de acesso aos recursos e envolvimento do aluno gerou a possibilidade de classificar os alunos em cinco outros subgrupos: 
Grupo A = 4 estudantes que realizaram mais de 15 interações semanais na plataforma;

Grupo B $=5$ estudantes que realizaram até 8 interações semanais na plataforma;

Grupo C $=10$ estudantes que realizaram até 5 interações semanais na plataforma;

Grupo D = 24 estudantes que não entraram regularmente na plataforma nem realizaram significativas atividades;

Grupo E $=6$ estudantes escolhidos para participar da plataforma durante o sorteio em sala, mas que nunca entraram na rede;

Como o objeto de estudo desta pesquisa concentra-se em entender os efeitos do artefato sobre o processo de aprendizagem dos alunos, foram escolhidos trabalhar com os dados de utilização dos grupos A, B e C porque demonstraram um uso efetivo da plataforma. É importante ainda ressaltar que tal classificação (A, B, C, D e E) foi realizada na primeira semana de operação do artefato (março de 2019) e sofreu alterações ao longo dos três meses de homologação do método proposto, visto que o interesse e acesso por parte dos alunos sofreu grande variação.

O resultado desse acompanhamento é apresentado na Tabela 8 que consolida os índices de acertos gerais para cada uma das quatro áreas apresentadas.

Tabela 2 - Evolução mensal dos índices de acertos da plataforma por grupo

\begin{tabular}{ccccc} 
& & Mar & Abr & Mai \\
\hline \multirow{3}{*}{ Grupo A } & L & $42,45 \%$ & $41,28 \%$ & $49,67 \%$ \\
& M & $31,71 \%$ & $34,22 \%$ & $36,15 \%$ \\
& CH & $39,63 \%$ & $41,82 \%$ & $43,05 \%$ \\
Grupo B & CN & $36,58 \%$ & $36,79 \%$ & $38,14 \%$ \\
\hline \multirow{3}{*}{ Grupo C } & L & $43,23 \%$ & $43,58 \%$ & $45,39 \%$ \\
& M & $30,93 \%$ & $31,16 \%$ & $32,58 \%$ \\
& $\mathrm{CH}$ & $38,36 \%$ & $39,15 \%$ & $40,17 \%$ \\
& $\mathrm{CN}$ & $37,65 \%$ & $39,42 \%$ & $40,04 \%$ \\
\hline & $\mathrm{L}$ & $40,89 \%$ & $39,14 \%$ & $40,29 \%$ \\
& $\mathrm{M}$ & $30,44 \%$ & $29,56 \%$ & $31,12 \%$ \\
& $\mathrm{CH}$ & $36,78 \%$ & $35,92 \%$ & $36,54 \%$ \\
\hline
\end{tabular}

L = Linguagens e códigos / M = Matemática / CH = Ciências Humanas / CN = Ciências da Natureza

Pode-se observar, que desde o início, os grupos que mais utilizaram o ambiente (grupos A e B) aprenderam mais que o grupo que menos a utilizou (grupo C). Em três meses de uso, o maior índice de aprendizado, que foi integralizado pela porcentagem de acertos de questões em cada uma das quatro áreas, majoritariamente está relacionado ao grupo que mais utilizou a ferramenta (grupo A), obtendo nas 4 áreas o maior percentual de aprendizagem. O Gráfico 1 representa visualmente essa evolução.

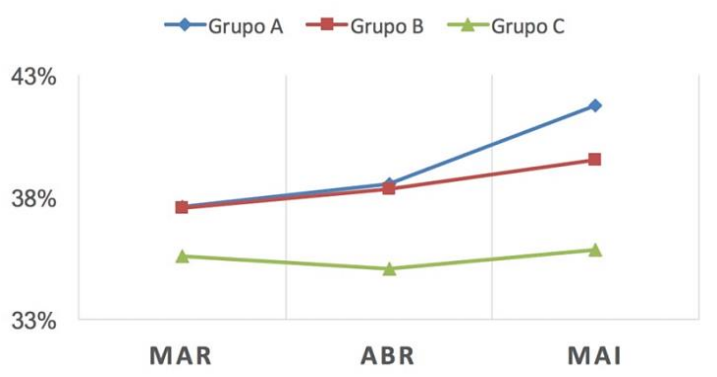

Gráfico 1 - Representação da evolução mensal dos índices de acertos da plataforma por grupo. Fonte: ARRAIS (2019). 
Foi realizada também uma pesquisa qualitativa com o grupo final de 43 alunos a fim de entender se eles utilizam outras formas de estudos independentes. O questionário teve um total de 14 respondentes sendo que: 1 aluno utiliza a plataforma e cursinho preparatório; 7 alunos utilizaram a plataforma dessa pesquisa e canais de vídeo do Youtube; 6 alunos utilizaram somente a plataforma dessa pesquisa. O questionário também perguntou aos alunos sobre a influência do uso do artefato proposto no processo de aprendizagem dos conteúdos do ENEM, e o retorno é unânime em afirmar que tem grande influência. Somente um aluno afirmou ter uma influência moderada.

\section{Conclusões}

A fase de avaliação da plataforma, que determinou o conjunto de resultados apresentados nessa pesquisa, demonstra que houveram ganhos de aprendizagem de quando o grupo de alunos iniciou o processo (março de 2019) até a fase final da pesquisa (junho de 2019). No resultado da fase de testes e validação do artefato foi possível observar que quanto maior o uso das atividades propostas no artefato, melhor o rendimento nos testes que aferem a proficiência. Os experimentos demonstram que o método pode promover a aprendizagem, mas para que seja eficiente é necessário o uso contínuo por um período de pelo menos 12 meses para que seus ganhos percentuais sejam mais expressivos.

Com base na fase de testes, simulados e questionários aplicados a alunos e professores, a pesquisa concluiu que o método apresenta ganhos de aprendizagem, mas, por limitações do processo de teste e do cronograma do estudo, não foi possível extrair resultados com alto grau de confiabilidade.

Como trabalhos futuros, o pesquisador gostaria de testar a aplicabilidade do método em cenários e ambientes diferentes, gerando alternativas derivadas desse processo. Outra necessidade observada é incluir no método uma camada de inteligência artificial, através de chat bots para ajudar alunos a encontrarem algum conteúdo de estudo, sem a necessidade de realizar as atividades da plataforma. Também é recomendável a aplicação de técnicas de otimização do artefato para que os processos executados pelos robôs autônomos sejam mais eficientes em seu processamento computacional.

\section{Referencias}

Araújo, R. (2016). "Sistemas de Informação e os desafios do mundo aberto. Seminário GranDSI-BR no SBSI 2016".

Araújo, R.M., Maciel, R.S., Boscarioli, C. (2017) "I GranDSI-BR: Grandes Desafios de Pesquisa em Sistemas de Informação no Brasil (2016-2026)" - RT. CE-SI da SBC. ISBN 978-85-7669-359-8. http://www2.sbc.org.br/ce-si//arquivos/grandsi.pdf.

Arrais, M. (2019) "Processamento de conteúdo, proficiências, deficiências e de interações sociais para a preparação para o Exame Nacional do Ensino Médio", Tese, Programa de Pós-graduação em Informática, UFRJ.

Dresch, A., Lacerda. P. D., Junior, A.V.A.J. (2015) “Design Science Research". Bookman. 
Hernández-García, Á., González-González, I., Jiménez-Zarco, A. I. and ChaparroPeláez, J. (2015). "Applying social learning analytics to message boards in online distance learning: A case study," Comput. Human Behav., vol. 47, pp. 68-80.

Kaiser, C. and Bodendorf, F. (2012). "Mining consumer dialog in online forums," Internet Res., vol. 22, no. 3, pp. 275-297.

Kang M., Kim, B., Gloor, P. and Bock, G.-W. (2011). "Understanding the effect of social networks on user behaviors in community-driven knowledge services," J. Am. Soc. Inf. Sci. Technol., vol. 62, no. 6, pp. 1066-1074.

Kaptein, R. and Kamps, J. (2011). "Explicit extraction of topical context," J. Am. Soc. Inf. Sci. Technol., vol. 62, no. 8, pp. 1548-1563.

Leginus, M. and Zhai, C. (2015). DOLOG, Peter. "Personalized generation of word clouds from tweets," J. Assoc. Inf. Sci. Technol., p. n/a-n/a.

Montejo-Ráez, A., Martínez-Cámara, E., Martín-Valdivia, M. T. and Ureña-López, L. A. (2014). "A knowledge-based approach for polarity classification in Twitter," J. Assoc. Inf. Sci. Technol., vol. 65, no. 2, pp. 414-425.

Moraes, A., Belluzzo, W. (2014). "O diferencial de desempenho escolar entre escolas públicas e privadas no Brasil", Nova Economia. vol.24 no.2 Belo Horizonte Mai/Ago.

Pasquali, L. (2003) "Psicometria: teoria dos testes na psicologia e na educação", Petrópolis, Vozes. 397 p.

Shapira, B. and Zabar, B. (2011). "Personalized search: Integrating collaboration and social networks," J. Am. Soc. Inf. Sci. Technol., vol. 62, no. 1, pp. 146-160.

Shi, C., Liu, J., Zhuang, F., Yu, P. S. and Wu, B. (2016). "Integrating heterogeneous information via flexible regularization framework for recommendation," Knowl. Inf. Syst., pp. 1-25.

Tang, L. and Liu, H. (2011). "Leveraging social media networks for classification," Data Min. Knowl. Discov., vol. 23, no. 3, pp. 447-478.

Vilares, D., Alonso, M. A. and Gómez-Rodríguez, C. (2015). "On the usefulness of lexical and syntactic processing in polarity classification of Twitter messages," J. Assoc. Inf. Sci. Technol., vol. 66, no. 9, pp. 1799-1816.

Yi, K. (2012). "Harnessing collective intelligence in social tagging using Delicious," J. Am. Soc. Inf. Sci. Technol., vol. 63, no. 12, pp. 2488-2502.

Zhou, B. and Pei, J. (2011). "Aggregate keyword search on large relational databases," Knowl. Inf. Syst., vol. 30, no. 2, pp. 283-318.

Zichermann, G. and Cunningham, C. (2011). "Gamification by Design: Implementing Game Mechanics in Web and Mobile Apps”, Canada: O’Reilly Media. 